\title{
Human-based solutions for open-door train departures
}

\author{
F. Jubert \\ Department of Safety Control, RATP, France
}

\begin{abstract}
From November 2005 to December 2012, an average of 6 trains per year departed with their doors open for the regional express railway lines exploited by the RATP in Paris. Aside from these general figures, the statistics do little to explain such incidents. For this reason, it is necessary to carry out a qualitative study in order to better understand this phenomenon.

Having examined all reports on the 46 open-door departures recorded over the analysis period, in addition to some driver interviews, the study succeeded in characterising the different types of errors that cause these incidents. The "split of double capture" that occurs when a driver shifts his attention to something other than the door service at the time of departure accounts for about $60 \%$ of the cases with known causes. The remaining $40 \%$ are caused by "omissions" following an unexpected interruption while carrying out the traveller service. Although the unawareness of focusing mechanisms makes it impossible to eliminate the problem, the conspicuous nature of the interruption allows drivers, when required, to implement two practices aimed at a better management of sequence breaks. The first consists of a "no interruptions" policy when launching the closing sequence. If the interruption is unavoidable however, the second practice will enforce a "start from scratch" policy when recommencing the door service. However, in order to be implemented, the latter practice assumes that the driver is doubtful over state of the door service, which is not always the case. The recorded incidents following sequence breaks attest to this.

On the basis of these different observations, the study sought to define the triggers likely to limit the occurrence of such incidents or, more specifically, to stem the production of driving errors that cause these incidents.
\end{abstract}

Keywords: railway safety, risk management, human performance practices. 


\section{Introduction}

The ability of a train conductor to start running his train without closing the doors constitutes a serious incident in terms of railway safety. The failure to close doors could cause one or more passengers to fall out of a moving train.

\subsection{Background}

Even though a technical solution for such incidents exists and is being implemented, through creating a connection between traction and the door closing control, this will not be installed on all operating vehicles for another few years. Until then, the transition period must be handled with care.

\subsection{Problem statement}

This work is intended to ensure this kind of safety, in hoping to learn more about driving errors and potentially identifying solutions that can be widely implemented. The aim is to analyse moving trains with doors open from various perspectives, by applying the behavioural sciences. In practical terms, our task involves interpreting the errors that cause incidents, in accordance with the present state of psychological knowledge. The findings should allow for a better understanding of the cognitive mechanisms at work when these errors occur and help determine appropriate solutions. The underlying notion is that preventive measures are only effective when they stem from an extensive study of causes.

\subsection{Investigation methodology}

To conduct this study, I began by obtaining all the reports on the 46 open-door train departures that occurred between November 2005 and December 2012 for the regional express railway lines exploited by the RATP in Paris. I then proceeded with an analysis of report details, principally the conductors' declarations. This information, recorded just after the incidents, enabled us to classify the sources of failure. Based on these results, I attempted to shed light on the different circumstances leading to such events.

\subsection{Presentation}

The in-depth causes of open-door departures will be exposed in the first part of this paper. The second part will present the solutions implemented by drivers to maximising the reliability of the door service. Finally, the study will offer possible paths towards reinforcing human reliability as regards door closing systems and critical driving operations in general.

\section{The causes of open-door departures}

After providing a detailed description of the door-closing sequence, the study will come back to the causes behind open-door departures. 


\subsection{Door-closing sequence}

The door-closing sequence is part of a wider series of actions known as the "traveller service". As the procedure indicates, this service has 4 phases:

1. Surveillance of the traveller exchange

2. Decision on the door closure

3. Door closure

4. Train departure

By referring to driving manuals on the various materials used on trains and on the procedures, the sequence from the door-closing action to the train's departure can be described in a precise manner.

In a normal situation, doors are closed by holding down the "General DoorClosing Button" (BP FP-G). This action sets off a buzzer at the end of which a "double beep" is sounded, indicating that the doors are checked and locked. On the signalling box of the control console, the "doors not locked" warning light then switches off indicating that a mechanical force has checked and locked the doors.
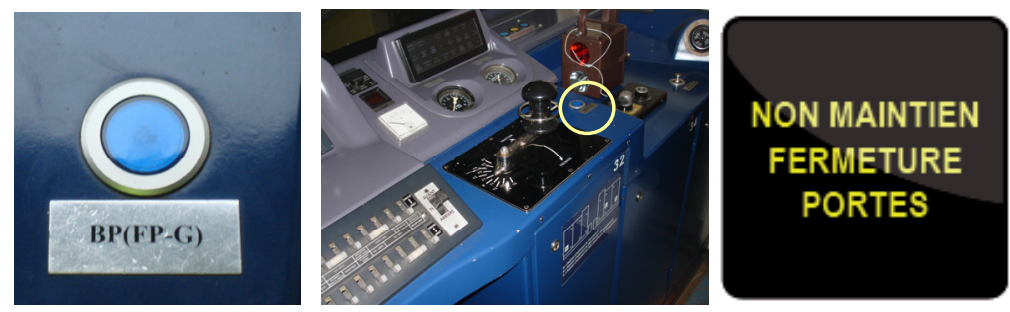

Figure 1: BP FP-G and "doors not locked" warning light.

When the "double beep" is heard, the driver must check that the door line is completely clear. Then, if there is no order, signal or circumstance, he can start the traction by moving the power handle forward to put his train in motion and leave the station.

These different elements enable an even more fine-tuned study that aims specifically at understanding what causes a driver to drive off without hearing the "double beep" and with the doors not locked light on.

\subsection{Types of errors detected}

These departures are caused by a lapse in the driver's attention during the traveller service. With respect to the analysis of incident reports, these "attention failures" come in two different forms. Nevertheless, the classification of incidents in one of the types of errors is strongly dependent on the statements collected, which have sometimes required interpretation. Choices made in this way may be arguable. These choices affect the number of incidents attributed to 
one type of error or another. However, they do not challenge the fact that opendoor train departures are caused by two types of errors.

In the interest of transparency, I have sought to make them visible to the reader, by integrating into the text various excerpts of statements which served as a basis for the classification.

\subsubsection{Omission following an interruption}

In $38 \%$ of the incidents in which the cause was known, these errors take the form of "omissions following interruption" [1] (12 cases). Concretely, the driver forgot to close the doors after an unexpected interruption. This failure is linked to an external occurrence that caused a break in the execution of the traveller service before the door-closing phase. Upon recommencing, the driver thought that the doors were closed and set the train in movement.

In the following incident report excerpts, the underlined elements are those which I considered to be a break in the traveller service:

"I can't believe how this kind of mistake could have happened, unless $\underline{a}$ driver in Torcy asked to get into the cab when the doors were closing. The door line being clear, I put the train in movement thinking they must have been closed."

"When I was about to start my door closing sequence, an agent in uniform signalled that he wanted to come aboard. At that moment I thought that I had closed the doors, and I left with them open."

"During the travel service, as I was getting ready to close my doors, I was interrupted by a traveller who was shouting and cursing me because there were no more stops at Mitry, and saying that I was the $5^{\text {th }}$ train going to Roissy."

\subsubsection{Split of double capture}

The remaining 59\% of errors are a result of "double capture split" [1] (19 cases). They are so named because these errors involve the simultaneous execution of several tasks (generally two), resulting in shared attention. It should be noted that constant attentional resources are required for a driver to properly perform his duties since several sets of information must be handled simultaneously. Moments in which attention is shared are not uncommon. However, such situations do not lead to errors that often. In fact, while the concomitance of tasks is a necessary condition, it is not sufficient to trigger an error. The driver's attention would also have to be "captured" by one task, to the detriment of the other. Given that the quantity of available attentional resources is in essence limited, beyond a certain required level, it becomes impossible to efficiently perform two tasks.

The same principle applies when a driver is called upon to manage another task in parallel to the traveller service and then focuses on this other task. In one of the interviews held, a driver commented on this by using the expression "to deal with another thing": "that's what happens when you deal with another thing!" His attentional resources are not focused at that moment on the door service when it should be carried out. The closing sequence gets omitted or is 
badly performed. To classify incidents in this error category, I sought, across various reports, elements attesting either:

- The management of a concomitant activity with the traveller service (elements underlined in the excerpts below);

- $\quad$ Or the presence of an activity requiring major involvement of the driver, and therefore, a significant demand on his attentional resources to the detriment of those which were focused on the traveller service.

Based on this, I was able to determine several attention-related incidents that altered the execution of the traveller service:

"I set my train in movement and (while conversing with the Regulations Officer), I realised that there was no double beep."

"I answered the Regulations Officer who asked me to drop off these blocs at Nation. I told him that I dropped them off at Vincennes at the end of the platform and at the same time I released the Immobilisation Brake. In my hurry, I forgot my door-closing sequence and I started the traction."

"In Vincennes, a station agent came into the cab to go to Val de Fontenay. When the agent got off the train, the driver was talking to him while launching the door-closing sequence. The driver didn't see that the doors weren't closed and he put his train in movement."

\subsubsection{Common factor}

In both types of errors, the drivers said that they were "sure" that they closed the doors and thus, heard the double beep. This is the reason why they did not check to see that the "doors not locked" light was switched off on the signalling box and subsequently drove off with it on. We may assume that hearing a double beep repeatedly at previous stations causes the sound to stay in the mind of the driver and thus replaces the beep that was not sounded, thereby reinforcing an incorrect perception of the situation.

\subsection{Identified causes}

These departures are caused either by a break in the execution of the traveller service, or when the driver's attention diverts to something other than the door service. Although the end-result is the same (an open-door departure), the process is different. On the one hand, attention is diverted. On the other, it gets divided. This is yet another more subtle difference which needs further examination in light of its implications for our work. It involves the awareness of what happens when an error occurs. While the driver may be aware of an interruption, he cannot perceive his own attentional capture. In other words, the break in sequence is directly perceptible by the driver. The interruption leaves a trace in the memory of the driver, which may or may not be used or not when he resumes the traveller service [2]. Conversely, the attention focusing gradually and unconsciously restrains one's field of attention as the mind concentrates on one thing alone [3]. This phenomenon is so insidious that it only enters the driver's consciousness after the fact and, again, only when a problem arises. 
Table 1: Comparison of the 2 causes of open-door departure.

\begin{tabular}{|c|c|c|}
\cline { 2 - 3 } \multicolumn{1}{c|}{} & Break in sequence & Attention focusing \\
\hline Origin & Unexpected interruption & Attentional capture \\
\hline Type of occurrence & Conscious & Unconscious \\
\hline $\begin{array}{c}\text { Perception level } \\
\text { during the } \\
\text { occurrence }\end{array}$ & Perceptible & Imperceptible \\
\hline
\end{tabular}

From the above table, we will see how these seemingly insignificant differences are in fact important in identifying the potential solutions to be put in place.

\section{Solutions implemented}

In addition to using technical devices, drivers carry out several practices aimed at maximising the reliability of the door service. The interviews I carried out have helped to identify two such practices related to the management of interruptions. The small number of interviews does not however provide an exhaustive overview of the solutions put in place to counter this risk.

\section{1 "No interruptions"}

When asked about the practices used for remembering to close the doors before driving off, the drivers unanimously said that from the moment the closing sequence started, it had to be completed. This is how the drivers sought to avoid interruptions once the door-closing sequence began. Some of them told us that the "no interruptions" rule after starting a sequence did not only apply to the door service but to all other critical activities in general. Based on the rule that $a$ job halfway done is a bad thing! the priority is given to completing the task in progress before beginning another. The objective is to avoid the potentially negative effects of an interruption while performing a task by focusing on the sequence. This rule can however become counter-productive in the event of an activity that takes priority over the door service if it is considered to be an emergency or to have potential consequences on the traveller service. In fact, if this new priority task cannot be carried out in parallel to the traveller service, the driver will have no other choice but to momentarily allow the interruption to deal with the situation. In such cases, drivers develop another complementary practice described in the paragraph below.

\section{2 "Start from scratch"}

"If someone interrupts me, I'll start over from the beginning"; "You stop, and you re-open the doors"; "You start from scratch!" These statements gathered from the interviews describe another practice undertaken by most of the drivers. It consists of starting over from the beginning of the traveller service in the event of an unexpected interruption. If, at the time of the interruption, the doors are 
closed, the driver can open the doors again if the interruption is prolonged. When he recommences, he will complete the entire traveller service.

This practice aims at removing all doubt over whether the doors are open or closed. In fact, with experience, the execution and sequencing of traveller service phases are mostly automated in the mind of the driver. Any break in this automation can throw the driver into incertitude over the phase reached at the time of the interruption. In other words, the driver knows that he was interrupted, but is less certain of where he stopped. Starting from scratch allows him to reinitialize the routine. In so doing, he can ensure that the doors are closed when he needs to put the train in movement.

\subsection{Limitations of these practices}

Among the four drivers interviewed, three had carried out an open-door departure. Given that these three are the same ones who described the abovementioned practices, it is legitimate to question the efficiency of these practices or, at least, the reason why they were not put into practice at the time of the incident.

First of all, it must be noted that of the three incidents, two were caused by "split of double capture". However, producing this type of error is not prevented by the above-mentioned practices. The reason is simple: because focusing attention is an unconscious phenomenon, the driver does not have any intelligible reason to use it.

The third incident is in itself, much more interesting in the sense that the error comes from an omission following an interruption. It happens when driver applies a strict application of the no interruptions rule until it becomes a never get interrupted rule. However, the first exception to this rule apparently caught him off-guard: "I don't know what happened. Normally I never stop, but that day..." One of the dangers of this practice, if applied in such an exclusive way, is that it does not allow the driver to use the reflexes necessary for managing an unexpected interruption. He may therefore find himself unprepared for such a situation.

Generally, the solution of starting from scratch in the event of an interruption is inefficient if the driver is convinced that he closed the doors. This is also the reason why a part of the incidents recorded over the study period occurred. In fact, this solution is only used if there is doubt over the state of the door service, which is not the case as seen in the statements gathered in the incident reports, since the drivers said that they were sure they had closed the doors.

I tried to take these different limitations into consideration when drafting my action proposals.

\section{Conclusion: prospects for action}

To conclude this work, I will discuss some hypothetical prospects for action. The proposed action plans are based on the principle that we can use an error to learn 
how to prevent it [4]. In other words, understanding the mechanism behind an error is a means for preventing and possibly correcting it.

\subsection{Sensitizing drivers on human errors}

The knowledge of mechanisms behind human errors has now advanced to the point where it is possible to transmit a certain quantity of information to those who bear the brunt of these failures. We train the drivers on the technical dysfunctions that could occur on their trains as well as how to handle them. So why not also inform them on their own propensity to making mistakes? They are, after all, the main actors involved. Moreover, the human error mechanisms proposed in this report are expressed in terms that are general enough to be understood by anyone. Integrating the elements of this study into the training offered to drivers, in the context of sensitization, could prove fruitful.

Additionally, this is a job that involves constant decision-making on priorities and often on when to stop (reflection in progress) to make resources available (reducing mental burden) as well as keeping other priorities in mind. Deciding among all these competing tasks can be crucial for railway safety. Shedding light on these internal decisions in the context of errors which a driver might commit can bring not only relief but also a sense of perspective on the importance of prioritizing tasks in the cab, particularly when managing communications.

\subsection{Reinforcing self-control}

Apart from the practices already developed to manage sequence breaks, which should be used and disseminated as widely as possible, two tools for ensuring reliability could reinforce a driver's self-control during the critical moments of an interruption. It involves treating with the interruption via two reliabilityoriented practices [5] consisting on the one hand of verbalising the state of the work situation right before interrupting a task and, on the other hand, of allowing oneself a "2-second stop" when recommencing the task. The idea is to use these practices to correct any flawed perception concerning the state of the door service when the driver recommences the traveller service following an interruption.

\subsubsection{Using existing reliability practices}

If drivers display a high level of reliability in the execution of the door service, it will have largely resulted from the reliability-oriented practices which they developed to manage unexpected interruptions during the traveller service. Trying to limit interruptions in progress on the one hand, and starting from scratch when such interruptions are inevitable on the other hand, constitute two good practices which should be acknowledged and shared, if not already done. However, they should not in any way be presented as "the" cure-all solution. Drivers must be aware of their limitations and avoid making exclusive interpretations. 


\subsubsection{Verbalising before interrupting}

Self-control is a professional act. It should not remain only in one's thoughts but should also be expressed in work practices. Verbalisation is one such practice. It consists of saying aloud what one is doing. This enables access to another level of the memory, that is to say, going from the immediate memory of about two seconds to the much longer lasting medium-term memory. In fact, making an oral statement allows it to be stored in the memory for about 10 minutes.

This practice is already being implemented on other networks in certain circumstances to ensure driving safety. Crossing a red signal, for example, requires a verbalisation by the driver regarding the state of the signal so as to activate his auditive memory as reinforcement to his already engaged visual memory.

In the case of interruptions in the execution of the traveller service, verbalising either the state of the door service or the last action carried out right before stopping would be a good way to limit incidents caused by a break in sequence. It is of course unnecessary to "verbalise" at each station stop, but rather only when interrupting a task. This oral statement allows a driver to memorize the state of the situation before the interruption, which becomes useful information when he recommences the traveller service. The objective is to leave a trace in the mind of the driver when he recommences.

\subsection{3 "2-second stop" before recommencing}

The moment of recommencing an interrupted activity is a particularly fertile breeding ground for human error. The incidents studied here provide the proof of this. Taking a few seconds to start over a quick check of the control console, the signalling box, and the state of the traveller service can be precious. The idea is to take a step back before acting to get a global picture of the situation and not to get caught up too quickly in the action. Right before recommencing, this "twosecond stop" requires a driver to extract himself from the urgency of the action in order to take a $360^{\circ}$ analytic glance at the driving environment. The goal is to avoid hurrying.

"Verbalizing the situation" before stopping and the "2-second stop" before recommencing comes from each driver's own initiative and must be valued by him. These practices cannot be imposed. In any case, it would be very difficult to monitor their application. That would assume a great effort on the part of the direct management to convince drivers that these practices are beneficial and to gain as much support as possible, without which these recommendations may go unheeded.

\subsection{Acting on sources of distraction}

The proposed courses of action until now do not concern the prevention of split attention failures, although these represent nearly $60 \%$ of errors leading to opendoor departures. The difficulty lies in acting on an error for which the mechanisms are unconscious. This unconscious state leaves little room for action. However, although we cannot counteract the problem, we can limit its sources. This also applies to the issue of sequence breaks. Reducing the source of 
the distraction such as an agent accompanying a driver in the cab is a first step in this direction.

\subsubsection{Cab access}

The first initiative consists of putting up a sign (see Fig. 2) on the cab door to dissuade unauthorized persons from entering. The objective here is to limit sequence breaks caused by someone entering the cab and the possible distractions, such as discussions, that this can create.

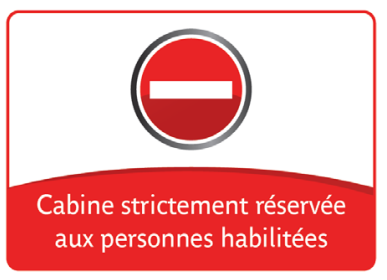

Figure 2: Sign-posting project.

\subsubsection{Cab behaviour rules}

Agents who are authorized to enter the cab must also be informed on the rules concerning behaviour once inside the cab, especially that of observing silence in the critical phases of driving. The idea is to limit disturbances during moments requiring the particularly close attention of the driver. A sticker reminding accompanying persons of the different situations where it is necessary to avoid verbal exchanges could be posted up in the cab (see Fig. 3).

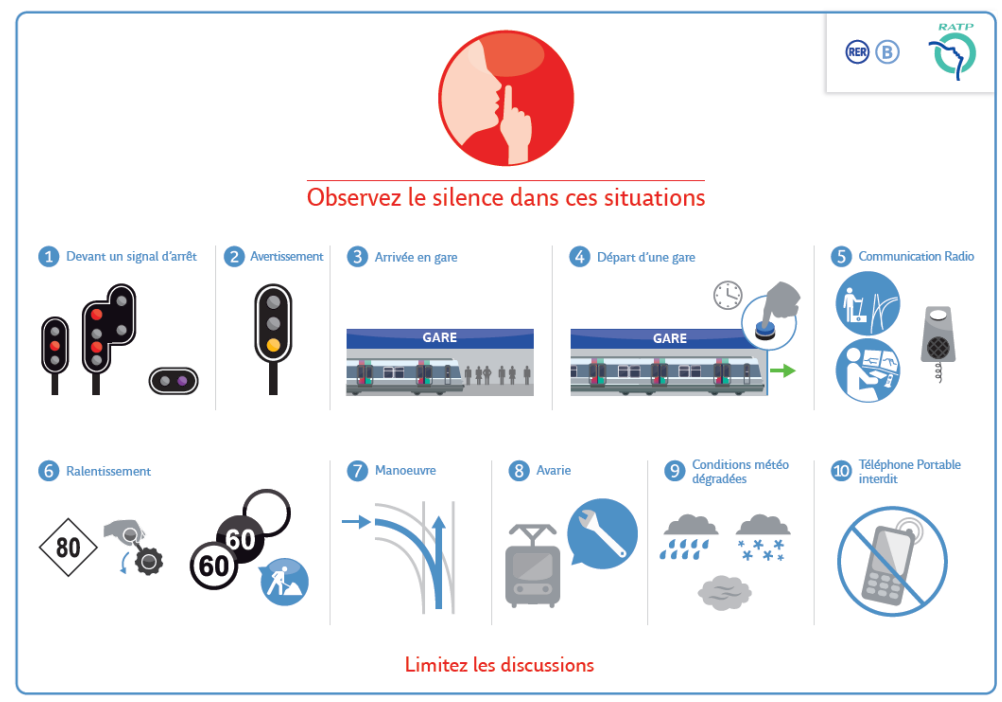

Figure 3: Sign-posting project in the cab. 
Additionally, the granting of any authorizations to enter cabs should be subject to a signed charter of good comportment. This commitment can be guided formally by the above-mentioned sign-posting project.

Lastly, the courses of action presented here are part of a global perspective of incident prevention linked to Human Factors. This is why efforts to agree on their implementation must necessarily be made in the context of their general benefits instead of focusing solely on open-door departures.

\section{References}

[1] Reason J., Human error, Cambridge University Press, Cambridge, 1990.

[2] Rasmussen J., Human errors: taxonomy for describing human malfunction in industrial installations, Journal of Occupational Accidents, 311-335, 1982.

[3] Lemaire P., Psychologie Cognitive, De Boeck, Bruxelles, 1999.

[4] Amalberti R., La conduite des systèmes à risques, Le Travail Humain, Paris, PUF, 1996.

[5] Theurier J.P., Le projet Performance Humaine, Document Edf, Direction Production Ingénierie, Division Production Nucléaire, 2010. 